\title{
Clinical Utility of Lefamulin: If Not Now, When?
}

\author{
Nicholas J. Mercuro ${ }^{1} \cdot$ Michael P. Veve ${ }^{2,3}$ (D) \\ Published online: 9 July 2020 \\ (C) Springer Science+Business Media, LLC, part of Springer Nature 2020
}

\begin{abstract}
Purpose of Review The looming threat of antimicrobial resistance requires robust stewardship and new developments in infectious diseases pharmacotherapy. This review discusses the pertinent spectrum and clinical data of lefamulin (Xenleta ${ }^{\circledR}$ ), with a focus on potential real-world use.

Recent Findings Lefamulin is a novel pleuromutilin antibiotic that obtained Food and Drug Administration labeling for community-acquired bacterial pneumonia (CABP) in 2019. Lefamulin is available in both intravenous and oral formulations, and it inhibits bacterial protein synthesis inhibition through interactive binding to unique sites of the peptidyl transferase center of the $50 \mathrm{~s}$ bacterial ribosome subunit. Resistance, including cross-resistance with other antibiotics, is infrequent. Lefamulin demonstrates activity against most Gram-positive pathogens and other organisms commonly associated with CABP, i.e., Streptococcus pneumoniae, Haemophilus influenzae, Mycoplasma pneumoniae, Legionella pneumophila, and Chlamydophila pneumoniae. Lefamulin may also be an option for serious public health threats like methicillin-resistant Staphylococcus aureus, vancomycinresistant Enterococcus faecium, and multi-drug-resistant organisms associated with sexually transmitted infections, e.g., Neisseria gonorrhoeae, Mycoplasma genitalium. Lefamulin lacks activity against Pseudomonas aeruginosa, Acinetobacter baumannii, Enterobacterales, most anaerobes, and E. faecalis. In Phase III trials, lefamulin monotherapy was non-inferior to moxifloxacin with or without linezolid for CABP.

Summary Lefamulin is a well-tolerated agent with a unique mechanism, availability in both IV and PO formulations, and it has been rigorously studied for safety and efficacy for CABP.
\end{abstract}

Keywords Pleuromutilin · Lefamulin · Treatment · Gram-positive $\cdot$ Methicillin-resistant Staphylococcus aureus · Antimicrobial stewardship

\section{Introduction}

Antimicrobial resistance is a significant public health threat, and multi-drug-resistant (MDR) pathogens are estimated to infect close to 3 million and kill more than 40,000 people in

This article is part of the Topical Collection on Antimicrobial Development and Drug Resistance

Michael P. Veve

mveve1@uthsc.edu

1 Department of Pharmacy, Beth-Israel Deaconess Medical Center, Boston, MA 02215, USA

2 Department of Clinical Pharmacy and Translational Science, College of Pharmacy, University of Tennessee Health Science Center, Knoxville, TN 37920, USA

3 Department of Pharmacy, University of Tennessee Medical Center, 1924 Alcoa Highway, Box \#117, Knoxville, TN 37920, USA the USA per year $[1,2]$. Strategies to curtail antimicrobial resistance threats are needed, and methods include improving appropriate antimicrobial utilization through stewardship and developing novel antimicrobial agents with activity against these concerning pathogens. While stewardship guidance and activities are growing [3, 4], investment in infectious diseases drug research and development $(\mathrm{R} \& \mathrm{D})$ has proved challenging due to regulatory barriers, historically poor development incentives, and limited uptake revenue [2]. Lefamulin (Xenleta $\left.{ }^{\circledR}\right)$ is a recently Food and Drug Administration (FDA)-approved, novel pleuromutilin antibiotic that demonstrates activity against common aerobic Gram-positive organisms, fastidious Gram-negatives, atypicals, and some Gram-positive anaerobes $[5,6]$, as well as resistant bacteria such as methicillin-resistant Staphylococcus aureus, vancomycin-resistant Enterococcus faecium, and MDR Neisseria gonorrhea [7, 8].

A concerning number of pharmaceutical companies have either sold off infectious diseases R\&D programs or filed for 
bankruptcy after a product launch [9]. These breakdowns are attributed to a misaligned health care reimbursement system and investment in the R\&D of "me too" agents, or drugs that offer little additional clinical benefit while having similar mechanisms and spectra of activity compared to existing antimicrobials $[9,10]$. In this aspect, lefamulin is distinct in that it displays a novel mechanism of action as well as activity against the more common types of bacteria that cause infections in the United States (USA), in addition to concerning Gram-positive organisms like methicillin-resistant Staphylococcus aureus (MRSA) that infect and kill more people than MDR Gram-negative organisms [11].

The pleuromutilin class was first developed in the 1950s, but no systemic agents have been available for human use until recently [12]. Lefamulin obtained an FDA indication for community-acquired bacterial pneumonia (CABP) in 2019 , is available in intravenous (IV) and oral (PO) formulations, and has a unique mechanism of action among systemic antimicrobial agents. In this review, we provide an overview of the structural activity relationship, spectrum of activity, and relevant clinical trial and safety data for lefamulin, with a focus on other clinical applications.

\section{Methods}

A systematic approach to literature search was performed on PubMed using the terms "lefamulin," "pleuromutilin," and "BC-3781," the investigational drug name for lefamulin. Results were limited to articles available in English and those describing systemic pleuromutilins used in human adults. Other clinical trials focusing on lefamulin were identified from clinicaltrials.gov, and additional data were obtained from pertinent conference proceedings and published abstracts related to lefamulin available through the manufacturer's webpage [13].

\section{Chemical Structure and Mechanism of Action}

Lefamulin is a semisynthetic derivative of the naturally occurring tricyclic diterpenoid pleuromutilin class of antibiotics. Pleuromutilins were first isolated from the fungus Pleurotus mutilus and eventually developed into systemic therapy for veterinary use and as a topical product for human use [5]. The tricyclic mutilin core of lefamulin contains a 14-carbon ring that is essential for antimicrobial activity, as this core interacts with the central portion of bacterial 23S rRNA through hydrophobic interactions, van der Waal forces, and hydrogen bonds. The modified $\mathrm{C} 14$ side chain, including a $\mathrm{C} 21$ keto group and additional R1 moieties, contributes to the physicochemical drug characteristics of lefamulin that distinguish it from other pleuromutilins (Fig. 1) [5]. This side chain consists of a thioether bond that allows for heightened solubility, potent antimicrobial activity, and metabolic stability that enables lefamulin to be administered in both IV and PO forms [5, 11]. The $\mathrm{C} 14$ side chain also helps lefamulin overcome bacterial ribosomal mutations and resistance development by maximizing the number of hydrogen bonds to the target site $[11,12]$.

Lefamulin inhibits bacterial protein synthesis through a unique mechanism of tight-fit binding to the $\mathrm{A}$ and $\mathrm{P}$ sites of the $50 \mathrm{~S}$ ribosomal subunit, creating an interference with peptidyl transferase to subsequently inhibit peptide bond formation and chain elongation $[11,14]$. Once elongation has started, lefamulin is ineffective $[5,11]$.

Development of resistance to this mechanism has been observed in vitro and in other pleuromutilins used in veterinary medicine, and it is often a result of various ribosomal protein point mutations within the domain $\mathrm{V}$ of the 23S rRNA that can affect the peptidyl transferase center (PTC) structure [15]. The most common resistance mechanisms via $23 \mathrm{~S}$ rRNA mutations include the $r p l C$ and $r p l D$ genes, which encode for ribosomal proteins $\mathrm{L} 3$ and L4. Mutations and substitutions in the $r p l C$ and $r p l D$ genes can cause conformational changes in the PTC and hinder correct positioning of the pleuromutilins in the tight-fit binding pocket within the $A$ and $P$ sites $[5,15]$. Other protein mutations, such as $v g a(\mathrm{~A}, \mathrm{~B} . \mathrm{E}), l s a(\mathrm{E})$, and $\operatorname{sal}(\mathrm{A})$, can confer pleuromutilin resistance via ribosomal protection [15]. Interestingly, intrinsic pleuromutilin resistance in Enterobacteriaceae is caused by the efflux of pleuromutilins mediated by the AcrAB-TolC efflux pump [5]. Real-world data describing lefamulin resistance via 23S rRNA target site mutations or cross-resistance to other ribosome inhibiting-antibiotics like lincosamides or oxazolidanones has been rarely observed $[5,12]$.

\section{Spectrum of Activity}

Lefamulin possesses a broad spectrum of activity against aerobic Gram-positive organisms. It has limited activity against Gramnegative pathogens, although it is active against fastidious Gramnegative organisms and atypical pathogens associated with CABP. Only three FDA breakpoints are in place for lefamulin susceptibility criteria: methicillin-susceptible $S$. aureus ( $\leq$ $0.25 \mu \mathrm{g} / \mathrm{mL})$, S. pneumoniae $(\leq 0.5 \mu \mathrm{g} / \mathrm{mL})$, and $H$. influenzae $(\leq 2 \mu \mathrm{g} / \mathrm{mL})$ [16]. Table 1 compares MIC distributions of lefamulin against other commonly used oral antimicrobials in the USA [18-32]. Global surveillance reports highlight lefamulin's in vitro activity against $S$. aureus; $E$. faecium; susceptible and drug-resistant $S$. pneumoniae, $H$. influenzae, M. catarrhalis, L. pneumophilia, M. pneumoniae, and $C$. pneumoniae; and various beta- and alpha-hemolytic streptococci [18]. The genes associated with vancomycin-resistance in E. faecium and $S$. aureus do not impact susceptibility to lefamulin $[15,18]$. Pleuromutilins also demonstrate activity against many common anaerobes like $C$. acnes, $C$. perfringens, 
Fig. 1 Chemical structure of lefamulin [11], pleuromutilins contain a 14-ring carbon mutilin core structure (right). A C-21 keto group and various $\mathrm{C}-14$ moieties (left) from a R1 side chain are responsible for the mechanism of action and spectrum of activity for lefamulin

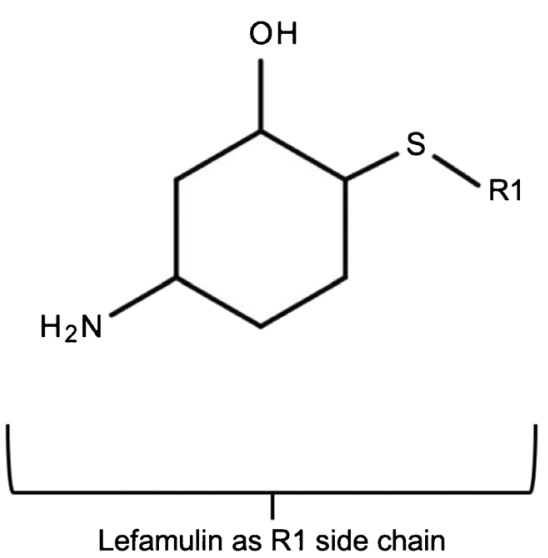

Fusobacterium sp. Peptostreptococcus sp., Prevotella sp., and sexually transmitted microbes, although no current FDA breakpoints exist for these organisms [16, 18]. The potential role against multi-drug-resistant enterococci and $N$. gonorrhoeae is enticing, although clinical data are lacking [7, 8, 18]. Notable gaps in antimicrobial coverage include most Enterobacterales, non-lactose-fermenting Gram-negative pathogens, E. faecalis, Clostridioides difficile, and Bacteroides sp. [18].

\section{Pharmacokinetics}

\section{General Pharmacokinetics of Lefamulin}

The FDA standard IV and PO lefamulin dosage is $150 \mathrm{mg}$ IV administered over $1 \mathrm{~h}$ every $12 \mathrm{~h}$ and $600 \mathrm{mg}$ PO every $12 \mathrm{~h}$, respectively [6]. Multiple Phase I trials were performed to assess lefamulin drug tolerability and pharmacokinetic profile in healthy volunteers. Intravenous lefamulin was found to be tolerable in up to $400 \mathrm{mg}$ in a dose-escalation study in healthy volunteers [31]. A pharmacokinetic study in healthy patients who received multiple doses of IV lefamulin $150 \mathrm{mg}$ every $12 \mathrm{~h}$ over a $1-\mathrm{h}$ infusion for 5 days found the mean (SD) total plasma maximum concentrations $\left(\mathrm{C}_{\max }\right)$ was $2.06 \mathrm{mg} / \mathrm{L}$ (0.737), with a mean (SD) half-life of $13.2 \mathrm{~h}(5.79)$ and displayed multiphasic decline [17]. The lefamulin package insert states the half-life of lefamulin is $8 \mathrm{~h}$ [6]. The mean (SD) total plasma area under the concentration-time curve over $24 \mathrm{~h}\left(\mathrm{AUC}_{0-24}\right)$ for the same IV dosing regimen was found to be $16.5 \mathrm{mg} \mathrm{h} / \mathrm{L}$ (6.21) [17]. A number of pharmacokinetic studies for the PO formulation found the dosage of $600 \mathrm{mg}$ every 12-h dosage achieved similar concentrations compared to $150 \mathrm{mg}$ IV every $12 \mathrm{~h}[6,33]$.

\section{Absorption}

In a two-part, open-label, crossover, randomized trial, 12 healthy adults received a single dose of PO immediaterelease lefamulin $600 \mathrm{mg}$ in either a fasted-state or after a high-fat meal [17]. Oral lefamulin was well tolerated, and the pharmacokinetics were similar to IV lefamulin $150 \mathrm{mg}$, but patients in the fed state had reductions in $\mathrm{AUC}_{0 \text {-inf }}(10 \%)$ and $\mathrm{C}_{\max }(28 \%)$ [17]. Lefamulin is subsequently recommended to be given $1 \mathrm{~h}$ before a meal or $2 \mathrm{~h}$ after a meal with water [6]. A multi-dose study included 8 healthy adults who received PO lefamulin $600 \mathrm{mg}$ every $12 \mathrm{~h}$ for 6 days and a single dose on day 7 , which was also well tolerated and displayed predictable pharmacokinetics (Table 1) [17]. The absolute oral bioavailability of lefamulin is $25.8 \%$ and $21.0 \%$ in fasted and fed states, respectively [17].
Table 1 Summary of steady-state pharmacokinetic data for $\mathrm{PO}$ and IV lefamulin [17]

\begin{tabular}{lllll}
\hline Administration & Dosage & $\mathrm{T}_{\max }$ (hours) & Total $\mathrm{C}_{\max }(\mathrm{mg} / \mathrm{L})$ & $\mathrm{AUC}_{0-24}(\mathrm{mg} \mathrm{h} / \mathrm{L})$ \\
\hline Oral, fasted & $600 \mathrm{mg}$ every $12 \mathrm{~h}^{\mathrm{a}}$ & $2.00(0.5-3)$ & $1.85(0.61)$ & $21.6(8.40)$ \\
Intravenous & $150 \mathrm{mg}$ after 5 days $^{\mathrm{b}}$ & - & $2.06(0.737)$ & $16.5(6.21)$ \\
\hline
\end{tabular}

All values are mean (SD), except median (range) is reported for $\mathrm{T}_{\max }$

${ }^{\text {a }}$ Parameters estimated after a 7-day course

${ }^{\mathrm{b}}$ Parameters estimated after a 5-day course 


\section{Distribution}

The volume of distribution of lefamulin is $86 \mathrm{~L}$, with plasma protein binding 95-97\% [6]. Lefamulin concentrations have been evaluated in pulmonary and skeletal/adipose tissues [34]. Pulmonary penetration was assessed in a pharmacokinetic study in 12 healthy men who received a single dose of lefamulin $150 \mathrm{mg}$ IV. Median (range) lefamulin $\mathrm{AUC}_{0-24} \mathrm{ex}-$ posures in epithelial lining fluid (8978 [no range provided] $\mathrm{ng} \mathrm{h} / \mathrm{mL}$ ) were comparable to total plasma levels (11,554 $\mathrm{ng} \mathrm{h} / \mathrm{mL}$ [1095-2343]) and considerably exceeded free plasma levels (1500.8 $\mathrm{ng} \mathrm{h} / \mathrm{mL}$ [10965-2343]). It is hypothesized that high pulmonary concentrations are due to the active transport of lefamulin into the alveolar-capillary membrane by P-glycoprotein [34]. Additionally, the results of a murine macrophage model determined that lefamulin is unaffected by lung surfactant [35]. These characteristics suggest lefamulin would have utility in treating bacterial pneumonia, as the site of infection is within the alveolar spaces and alveolar lining fluid within the interstitium of the lung [36]. Antibiotic penetration into pulmonary tissue and bronchial secretions is also dependent on several drug-related characteristics, such as molecular weight, low protein binding, and other structural characteristics [36]. A separate pharmacokinetic study performed in healthy volunteers determined that median (range) lefamulin $\mathrm{AUC}_{0-24}$ concentrations in skeletal muscle (1264.2 $\mathrm{ng} \mathrm{h} / \mathrm{mL}$ [770-2094]) and adipose tissue (1456.6 $\mathrm{ng} \mathrm{h} / \mathrm{mL}$ [810-1758]) were similar to free lefamulin concentrations in plasma, after a single dose of lefamulin $150 \mathrm{mg}$ IV administered over $1 \mathrm{~h}$ [34].

\section{Metabolism}

Lefamulin is hepatically metabolized by the hepatic microsomal P450 system, and predominantly the CYP 3A4 pathway. In vitro studies involving with human recombinant CYP450 enzymes established that lefamulin is metabolized by CYP3A as both a substrate and inhibitor [37]. Other studies did not reveal any significant induction of CYP1A2 [37]. Additionally, in vitro evaluation demonstrated that lefamulin is a P-glycoprotein substrate and a weak inhibitor of Pglycoprotein-mediated efflux transport [38].

In one study, PO rifampin $600 \mathrm{mg}$, a potent CYP3A4 inducer, reduced the mean lefamulin $\mathrm{AUC}_{0 \text {-inf }}$ and $C_{\max }$ by $28 \%$ and $8 \%$, respectively, when administered concomitantly with a single dose of IV lefamulin $150 \mathrm{mg}$. A subsequent study found that $\mathrm{PO}$ rifampin $600 \mathrm{mg}$ reduced the mean lefamulin $\mathrm{AUC}_{0 \text {-inf }}$ and $C_{\max }$ by $72 \%$ and $57 \%$, respectively, when concomitantly administered with a single dose of PO lefamulin $600 \mathrm{mg}$ [38]. PO ketoconazole $200 \mathrm{mg}$ twice daily, a strong CYP3A4 inhibitor, increased mean lefamulin $\mathrm{AUC}_{0 \text {-inf }}$ and $C_{\max }$ by $31 \%$ and $6 \%$, respectively, when administered concomitantly with a single dose of IV lefamulin $150 \mathrm{mg}$. A separate study found the same dosage of ketoconazole increased the lefamulin $\mathrm{AUC}_{0 \text {-inf }}$ and $C_{\max }$ by $165 \%$ and $58 \%$ when administered with a single dose of PO lefamulin $400 \mathrm{mg}$ [38]. Because of potential efficacy loss due to low exposure of LEF, co-administration of LEF with moderate and strong CYP3A4 or P-glycoprotein inducers should be avoided [6].

No clinically significant differences in the pharmacokinetics of PO digoxin $0.5 \mathrm{mg}$, a P-glycoprotein substrate, were observed when administered concomitantly with a single dose of lefamulin $600 \mathrm{mg}$ [38]. Lefamulin increased the arithmetic mean $C_{\max }$ and $\mathrm{AUC}_{0 \text {-inf }}$ of PO midazolam, a CYP3A4 substrate by approximately $100 \%$ and $200 \%$, respectively, when it was administered at 0,2 , or $4 \mathrm{~h}$ after administration of a single dose of PO lefamulin $600 \mathrm{mg}$ [38]. Based on animal, clinical trial, and other adverse drug events (ADEs) identified from Phase I-III trials related to QTc prolongation, the concomitant administration of lefamulin with other CYP3A4 substrates that prolong the QTc interval is contraindicated [6, 38]. Lefamulin prolongs the QT interval in a nonlinear, concentration-dependent manner via human-ether-a-go-gomediated potassium channel current interactions, and data from two Phase III trials found that the mean placebocorrected changes in QTc from baseline were $13.6 \mathrm{~ms}$ and $9.3 \mathrm{~ms}$ following administration of lefamulin $150 \mathrm{mg}$ IV every $12 \mathrm{~h}$ and lefamulin $600 \mathrm{mg}$ PO every $12 \mathrm{~h}$, respectively [6, 38]. Analysis of all post-baseline QTc values showed the proportions of subjects exposed to lefamulin or moxifloxacin had similar degrees of QT prolongation at day 3 or 4 of the studies; $17.9 \%$ of lefamulin subjects and $22.3 \%$ of moxifloxacin subjects had a mean change in QTc from baseline of more than $30 \mathrm{~ms}[38]$.

\section{Excretion}

In a non-pigmented rat model, lefamulin was excreted as $80 \%$ unchanged drug in feces and 13\% in urine [39]. Additionally, no clinically significant differences in the pharmacokinetics of IV lefamulin $150 \mathrm{mg}$ over $1 \mathrm{~h}$ for one dose were observed in 7 human subjects with normal renal function compared to 8 subjects with severe chronic renal impairment and 8 subjects who were receiving hemodialysis [40]. Specifically, the median (range) $\mathrm{C}_{\max }$ and $\mathrm{AUC}_{0 \text {-inf }}$ of subject with normal function was $3182 \mathrm{ng} / \mathrm{mL}$ (697) and $9004 \mathrm{~h} \mathrm{ng} / \mathrm{mL}$ (2591), respectively, compared to $3138 \mathrm{ng} / \mathrm{mL}$ (990) and 12,262 h ng/mL (7798) in patients with severe renal disease, and $3341 \mathrm{ng} / \mathrm{mL}$ (916) and $8955 \mathrm{~h} \mathrm{ng} / \mathrm{mL}$ (3103) in patients on hemodialysis [40]. No dose reduction recommendations are available for patients with renal dysfunction [6]. Lefamulin pharmacokinetics were also studied in un-infected volunteers with normal hepatic function, moderate (Child-Pugh Class B), and severe (Child-Pugh Class C) hepatic impairment, as a single dose of IV lefamulin $150 \mathrm{mg}$ given over $1 \mathrm{~h} \mathrm{[6]}$. The half-life of lefamulin was found to be prolonged in patients with Child-Pugh Class $\mathrm{C}$ hepatic 
impairment compared to normal hepatic function, with a mean (SD) $\mathrm{t}_{1 / 2}$ of $17.6 \mathrm{~h}$ (3.4) compared to $11.5 \mathrm{~h}$ (1.8). Unbound lefamulin plasma $\mathrm{AUC}_{0 \text {-inf }}$ was increased 3 -fold in subjects with severe hepatic impairment compared to that in subjects with normal hepatic function, due to the high protein binding observed in lefamulin. Based on these data, a dose reduction to lefamulin $150 \mathrm{mg}$ IV every $24 \mathrm{~h}$ is recommended in patients with Child-Pugh Class $\mathrm{C}$ hepatic impairment. No hepatic impairment data are available for lefamulin PO, and this formulation is not recommended for patients with Child-Pugh Class B or C hepatic impairment [6].

\section{Pharmacodynamics}

The 24-h free-drug area-under-curve (AUC) to minimal inhibitory concentration (MIC) ratio, or $f \mathrm{AUC}_{0-24} / \mathrm{MIC}$, has been shown to be the pharmacodynamic index associated with the antibacterial activity of lefamulin [41]. Lefamulin displayed a moderate post-antibiotic effect against $S$. pneumoniae and $S$. aureus in a neutropenic mouse model [41]. In the same study, lefamulin achieved 3- to 6-fold higher potency in the murine lung model when compared to the thigh model, consistent with pharmacokinetic data suggesting lefamulin achieves high pulmonary concentrations [41]. In a separate neutropenic murine lung and thigh study, the lefamulin $f \mathrm{AUC}_{0-24} / \mathrm{MIC}$ required for stasis was 8-fold lower in the lung model when compared to the thigh model [42]. An additional neutropenic murine thigh model determined plasma $f \mathrm{AUC}_{0-24} / \mathrm{MIC}$ ratios of 1.37 and 2.15 resulted in a $1-$ and 2$\log _{10}$ CFU reductions, which corresponded with ELF $\mathrm{AUC}_{0}$ ${ }_{24} / \mathrm{MIC}$ ratios of 21.7 and 63.9 [43].

Target attainment analyses for IV and PO lefamulin dosage selection in CABP were calculated using population pharmacokinetic models from Phase I/II data and from pharmacokinetic-pharmacodynamic targets associated with efficacy in S. pneumoniae and S. aureus [33]. Monte Carlo simulation was performed for IV lefamulin $150 \mathrm{mg}$ every $12 \mathrm{~h}$ and lefamulin $600 \mathrm{mg}$ PO every $12 \mathrm{~h}$ [33]. The probability of target attainment (PTA) was calculated for the total-drug ELF AUC/MIC and free-drug plasma AUC/MIC ratio targets associated with a $1-$ and $2-\log 10$ cfu reduction from baseline for both $S$. pneumoniae and S. aureus. The PTA in ELF for a 1$\log 10$ cfu reduction from baseline in $S$. pneumoniae ranged from 99.2 to $100 \%$ for IV and all PO regimens at the $\mathrm{MIC}_{90}$ $(0.12 \mathrm{mg} / \mathrm{L})$. In another $S$. pneumoniae simulation at the $\mathrm{MIC}_{99}(0.25 \mathrm{mg} / \mathrm{L})$ and for the same target $\log 10$ cfu reduction, PTA was $96.7 \%, 82.1 \%$, and $96.3 \%$ for IV and both fed and fasted PO-dosing regimens, respectively. The PTA for the total-drug ELF AUC/MIC ratio target associated with a 2$\log _{10}$ cfu reduction from baseline was also calculated for $S$. pneumoniae at the $\mathrm{MIC}_{90}$, and the PTA for IV and PO dosing regimens under fed and fasted conditions was $99.2 \%$,
$92.3 \%$, and $99.2 \%$, respectively. For the free-drug plasma AUC/MIC ratio target associated with either 1 - or $2-\log 10$ cfu reduction, percentage probabilities were $100 \%$ for each dosing regimen at $\mathrm{MIC}_{90}$ or $\mathrm{MIC}_{99}$ values. Similar PTA analyses were performed for $S$. aureus, and for the total-drug ELF, AUC/MIC ratio target for a $1-\log _{10}$ cfu reduction from baseline was within a range of 92.7 to $99.3 \%$ all dosing regiments at the $\mathrm{MIC}_{90}$ value $(0.12 \mathrm{mg} / \mathrm{L})$. To achieve a $2 \log _{10}$ cfu reduction from baseline for $S$. aureus at the MIC value of $0.03 \mathrm{mg} / \mathrm{L}$, and the PTA ranged from 98.4 to $100 \%$ for all dosing regimens. For the free-drug plasma AUC/MIC ratio target associated with either endpoint and a $\mathrm{MIC}_{90}$ or value of $0.25 \mathrm{mg} / \mathrm{L}$, percentage probabilities were $\geq 99.5 \%$ for each dosing regimen [33].

A population pharmacokinetic model was also performed for PO lefamulin $600 \mathrm{mg}$ every $12 \mathrm{~h}$ in fasted and fed states [44]. The percentage probabilities of target attainment, associated with a $1-\log ^{10} \mathrm{CFU}$ reduction for $S$. aureus and S. pneumoniae, were $100 \%$ at the $\mathrm{MIC}_{90}$ and $95.5 \%$ or higher at the $\mathrm{MIC}_{99}$ [33]. For $f \mathrm{AUC}_{0-24} / \mathrm{MIC}$ targets associated with a 2- $\log ^{10} \mathrm{CFU}$ reduction, percentage probabilities were $91.9 \%$ or higher at $\mathrm{MIC}_{90}$ values. Overall percentage probabilities of attaining $f \mathrm{AUC}_{0-24} / \mathrm{MIC}$ targets for 1 - and $2-\log ^{10} \mathrm{CFU}$ reductions of S. pneumoniae and S. aureus were 99.4 and $91.7 \%$ or higher, respectively, and the probabilities of target attainment were similar under fasted and fed conditions. A more indepth pharmacokinetic and pharmacodynamic review of lefamulin is published elsewhere [45].

\section{Summary of Clinical Trial Data}

\section{Community-Acquired Bacterial Pneumonia}

With potent activity against CABP pathogens and high pulmonary concentrations, lefamulin demonstrates antimicrobial properties ideal for respiratory tract infections. The Lefamulin Evaluation Against Pneumonia (LEAP)-1 and -2 studies were similarly designed multicenter randomized controlled trials that tested non-inferiority of lefamulin against moxifloxacin in adults with CABP $[46,47]$. Study details and comparisons of population characteristics and endpoints are in Tables 2 and 3. Early clinical response (ECR) was the FDA primary endpoint, defined as the response rate in the intent-to-treat (ITT) population at $96 \mathrm{~h}$ after the first study drug dose. "Responders" had $>1$ signs of clinical improvement and no worsening CABP symptoms. The FDA endpoint was evaluated using $12.5 \%$ and $10 \%$ non-inferiority margins in LEAP-1 and LEAP-2 studies, respectively. The European Medicines Agency (EMA) co-primary endpoint, investigator assessment of clinical response (IACR), was resolution of signs and symptoms, without need for additional antimicrobial assessed 5-10 days after the last dose of study drug. In the primary and 


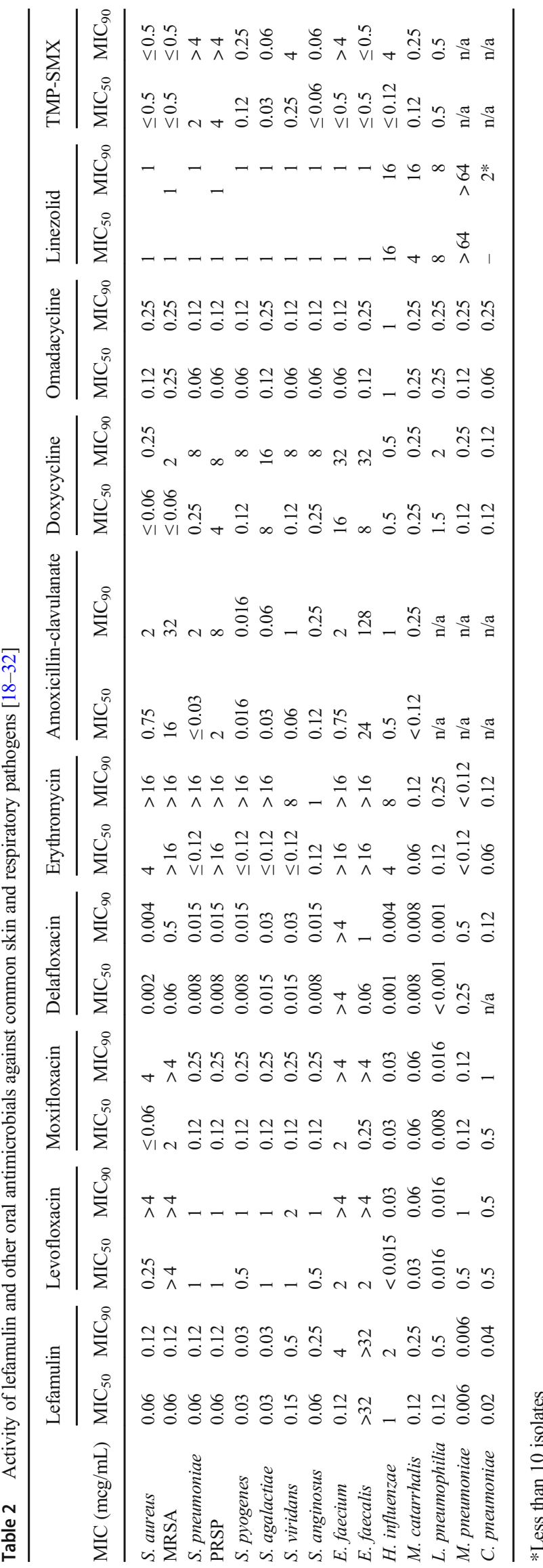

co-primary endpoints, lefamulin was found non-inferior to moxifloxacin in both trials. The most common organism identified in each study was $S$. pneumoniae, and while lefamulin maintains potent activity against MRSA, those who were at risk of developing MRSA pneumonia were unfortunately excluded from the LEAP-2 trial, and few patients from both trials combined had MRSA isolated. Most patients had Pneumonia Patient Outcomes Research Team (PORT) risk class $\leq$ III in both studies, or less severe pneumonia.

The LEAP-1 trial evaluated ECR with IV/PO lefamulin compared to IV/PO moxifloxacin \pm linezolid [46]. If MRSA was suspected at the initial screening, patients randomized to moxifloxacin also received adjunctive linezolid $600 \mathrm{mg}$ IV every $12 \mathrm{~h}$, and patients randomized to lefamulin received additional placebo. Patients were eligible to switch to PO therapy after receiving at least six IV doses, were hemodynamically stable and afebrile for $24 \mathrm{~h}$, had improving pneumonia symptoms, and had a functioning gastrointestinal tract. The populations were well balanced, although fewer patients < 65 years of age were in the lefamulin arm (52\% vs $61 \%$ ). The median duration of therapy was 7 days for both study drugs, and 38 and $44 \%$ of lefamulin and moxifloxacin courses, respectively, were switched from IV to PO. The overall rates of ECR ( $87 \%$ vs $90 \%$ ) and IACR ( $82 \%$ vs $84 \%$ ) were similar. In subgroup analysis, moxifloxacin had more favorable ECR in patients with $>2$ American Thoracic Society (ATS) severity criteria, i.e., confusion, uremia, respiratory rate $\geq 30$ breaths/min, hypotension, $\mathrm{PaO} 2 / \mathrm{FiO} 2 \leq 250 \mathrm{mmHg}$, multi-lobar infiltrates, leucopenia, thrombocytopenia, or hypothermia, $(76 \%$ vs $94 \%)$, and $<65$ years of age $(85 \%$ vs $93 \%$ ). The differences in ECR in younger patients was determined to be confounded by the presence of minor ATS criteria, and no specific ATS variables were found to be driving these differences in logistic regression analyses [LEAP-1]. In the microbiologic intent to treat-1 group, the most commonly isolated organisms were S. pneumoniae (60\%), H. influenzae (34\%), and atypical pathogens (29\%), and no MRSA was isolated. Of 6 lefamulin-treated patients with pneumococcal bacteremia, 1 achieved treatment success, while 5 were treatment failures for IACR at test of cure (TOC). Gastrointestinal disturbances such as diarrhea were more frequent in the moxifloxacin arm (1\% vs $8 \%$ ) while there was more infusion site pain in the lefamulin arm $(3 \%$ vs $0 \%)$.

LEAP-2 evaluated similar endpoints of ECR and IACR, except only using fixed durations of oral formulations for lefamulin ( 5 days) and moxifloxacin ( 7 days) in both inpatient and outpatient settings and a non-inferiority margin of $10 \%$ for both FDA and EMA endpoints [47]. A major difference in study design from LEAP-1 and LEAP-2 was the focus on the use of PO agents only without the addition of linezolid in the moxifloxacin group. Additionally, fewer patients in the LEAP-2 study had severe pneumonia as characterized by the 
Table 3 Summary of Lefamulin Evaluation Against Pneumonia (LEAP) trial data [46, 47]

LEAP-1 $(n=551)$

LEAP-2 $(n=738)$

Treatment assignments

Patients

Notable exclusions

Enrollment location

- Eastern Europe

- Latin America

- Asia and Africa

- N. America

PORT class

I

II

III

IV

$\mathrm{V}$

CURB-65

0

1

2

3

4

5

Pathogen identified

- S. pneumoniae

- S. aureus

- H. influenzae

- M. catarrhalis

- M. pneumoniae

- L. pneumophila

- C. pneumoniae

Early clinical response

Clinical response (modified ITT)

Test of cure, clinically evaluable

Treatment-emergent adverse events leading to death

Adverse drug event

- Diarrhea

- Nausea/vomiting

- QTc prolongation

Lefamulin $150 \mathrm{mg}$ IV Q12H with PO transition vs moxifloxacin $400 \mathrm{mg}$ IV Q24H, option to add linezolid for suspected MRSA; both groups able to treat up to 10 days

Clinical, laboratory, radiographic findings of pneumonia

- Receipt of non-study antibiotics

- Hospitalized 2 days or more in the last 90 days

- Confirmed/suspected for MRSA, Pseudomonas, resistant pathogens

- Attributable etiologies other than CABP (VAP, HAP, aspiration, etc)

- Resided in long-term care within 30-days of symptom onset

- Immunosuppression

- PORT I (least severe) or V (most severe)

- Severe hepatic, renal, cardiac, or hematologic disease

$78.9 \%$

$2.5 \%$

not specified

$0.5 \%$

$0 \%$

$0.2 \%$

$72.0 \%$

$26.5 \%$

$1.3 \%$

$10.3 \%$

$45.6 \%$

$35.4 \%$

$8.0 \%$

$0.7 \%$

$-$

318 (57.7)

197 (35.8)

$14(2.5)$

108 (19.6)

$36(6.5)$

$39(7.1)$

$32(5.8)$

$30(5.4)$

$87.3 \%$ vs $90.2 \%$

$88.2 \%$ vs $93.8 \%$

$86.9 \%$ vs $89.4 \%$

$2.2 \%$ vs $1.8 \%$

Lefamulin

$38.1 \%$

$0.7 \%$

$2.9 \%(+13.8 \mathrm{~ms})$
Lefamulin $600 \mathrm{mg}$ PO BID for 5 days versus 7 days moxifloxacin $400 \mathrm{mg}$ PO daily

Clinical, laboratory, radiographic findings of pneumonia 
PORT score and CURB-65 when compared to the LEAP-1 trial. ECR in both groups were $90.8 \%$, and there were no differences in microbiologic outcomes or in other subgroups. Similar to the LEAP-1 trial, the most commonly isolated organisms from LEAP-2 were $S$. pneumoniae (57\%), followed by $H$. influenzae (24\%). MRSA was isolated in 3 patients overall, but no notable outcome differences were noted by organism. In the lefamulin arm, 6 patients had bacteremia and four had baseline pathogens covered by lefamulin (3 with $S$. pneumoniae and 1 with $S$. aureus). Two of these patients achieved ECR and had an outcome of investigator assessment of clinical response success at TOC. The LEAP-1 and LEAP2 trials concluded that lefamulin is an effective option for CABP. While the short-term adverse effect profile was comparable to that of moxifloxacin, rare adverse drug events and long-term safety outcomes have not been identified.

\section{Acute Bacterial Skin and Skin Structure Infection}

A Phase II trial was performed to compare the efficacy of IV lefamulin to IV vancomycin in adults with acute bacterial skin and skin structure infection (ABSSSI) [48]. ABSSSI was defined as a wound, burn, cellulitis of $\geq 10 \mathrm{~cm}$ in size, erysipelas, or an abscess with cellulitis where the diameter of any abscess being $<75 \%$ of maximum dimension of the cellulitis; patients were required to have at least two signs of systemic infection related to ABSSSI. Patients were randomized to receive lefamulin $100 \mathrm{mg}(n=70)$, lefamulin $150 \mathrm{mg}(n=71)$, or vancomycin $1000 \mathrm{mg}(n=66)$, every $12 \mathrm{~h}$ for a duration of 514 days. The primary end point was clinical success rate at the test of cure (TOC) at 7 to 14 days after treatment was completed. and was categorized to success, improved, failure, or not evaluable.

The patient populations were similar. During the TOC visit to assess clinical success, $92 \%$ of vancomycin patients responded compared to $89 \%$ of the lefamulin patients receiving the approved FDA dose of $150 \mathrm{mg}$ every $12 \mathrm{~h}$. Most microbiologically evaluable infections were caused by $S$. aureus, and clinical success rates for lefamulin against PVL-positive and USA300 MRSA strains were similar or numerically higher than the corresponding clinical success rates for vancomycin [48]. As of the first quarter of 2020, no known Phase III trials have been developed further exploration of lefamulin for ABSSSI [13].

\section{Safety, Pregnancy, and Lactation}

No significant differences in lefamulin pharmacokinetics were observed in women or men, or patients across different age strata. A randomized, placebo-controlled trial was performed to investigate the safety, tolerability, and pharmacokinetics of IV lefamulin $150 \mathrm{mg}$ in 12 healthy men and 12 healthy women aged $18-55$ years, and also to 12 healthy elderly subjects $\geq$
65 years [49]. No clinically significant adverse events were observed in any subject.

From the LEAP-1 clinical trial data, infusion-site reactions occurred in $7 \%$ of patients treated with IV lefamulin and in $3 \%$ of those treated with IV moxifloxacin [46]. Diarrhea was less common with IV lefamulin compared to moxifloxacin (1 vs $8 \%$ ), yet more common in LEAP-2 with the oral formulation ( $12 \%$ vs $1 \%$ ), The rationale for this large difference is currently unknown. Hepatic enzyme elevations, nausea, hypokalemia, insomnia, and headache occurred with both formulations of lefamulin at rates similar to those with moxifloxacin [47]. In the Phase II ABSSSI trial, more headache, nausea, infusion site phlebitis, and pruritus were observed in the vancomycin arm when compared to the lefamulin arms, although statistical significance was not noted [48]. There was a higher incidence of QTc interval prolongation in the in the lefamulin arms compared to the vancomycin arms, none of which were prolonged $>480 \mathrm{~ms}$ or increased $>60 \mathrm{~ms}$ from baseline.

In animal studies, administration of lefamulin during pregnancy was associated with fetal loss, stillbirth, and decreased fetal body weight, and ossification. According to the label, women who could become pregnant should use effective contraception while taking lefamulin and for 2 days after stopping it. In rats, there were no effects on male fertility that were considered to be related to lefamulin [6]. Lefamulin concentrations have been detected in the milk of lactating rats, but no human lactation data are available [6]. Given potential drugrelated risks, the lefamulin package insert recommends that mothers who breastfeed should discard breast milk during lefamulin treatment and for 2 days after taking the last dose.

\section{Potential Clinical Application of Lefamulin}

\section{Lefamulin Use in Multi-Drug-Resistant Organisms}

\section{Methicillin-Resistant Staphylococcus aureus}

Methicillin-resistant $S$. aureus remains a significant cause of hospital- and community-acquired infections, ranging from minor acute bacterial skin and skin structure infections to more severe diseases including, but not limited to, bacteremia, osteoarticular disease, and infective endocarditis [50]. Despite medical advancements, invasive MRSA infections remain associated with high morbidity and mortality and are more prevalent than the sum of all health care-associated MDR Gramnegative bacilli infections in the USA [1]. In 2017, the CDC estimated 323,700 cases of invasive MRSA infections, resulting in 10,600 deaths [1]. Furthermore, the USA opioid epidemic has significantly contributed to injection drug-userelated staphylococcal infections [51].

While not clinically studied in severe MRSA infections, lefamulin has demonstrated activity against MRSA from 
surveillance data, with reported a $\mathrm{MIC}_{90}$ of 0.12 [18]. In a Phase II skin soft tissue infection trial, the most common organism isolated was $S$. aureus, with a high frequency of methicillin resistance and Panton-Valentine leukocidin (PVL) positivity [48]; other trials have not evaluated the presence of toxin production from clinical $S$. aureus isolates. Lefamulin was also found to have superior efficacy compared to vancomycin or linezolid in a murine $S$. aureus peritonitis-induced bacteremia model that included MRSA [52], and lefamulin also displayed a superior reduction in bacterial burden when compared to linezolid and tigecycline against MSSA, with comparable efficacy to daptomycin or vancomycin [53]. Other areas with potential clinical application but lacking data include prosthetic joint infections, osteomyelitis, periorbital cellulitis, or oral chronic suppressive therapy.

The current infectious diseases landscape focuses on PO antibiotics for the management of invasive infections to reduce central line complications and streamline transitions of care. This concept was recently demonstrated in the Oral versus Intravenous Antibiotics for Bone and Joint Infection (OVIVA) randomized trial where non-inferiority of PO stepdown was established for bone and joint infections [54]. Clinicians and investigators are becoming more comfortable transitioning patients with uncomplicated $S$. aureus bloodstream infections to PO regimens [55-57]. Regardless, lefamulin bone/joint concentration data and clinical evidence for patients with bloodstream infection are not available. While there are multiple available oral antibiotics with activity against MRSA, i.e., trimethoprim-sulfamethoxazole, linezolid, fluoroquinolones, most are associated with notable toxicities and intolerances, which ultimately could define a role for lefamulin.

\section{Vancomycin-Resistant Enterococcus faecium}

Infections due to vancomycin-resistant $E$. faecium are a continued threat given the limited number of antimicrobial agents with activity against this organism, and the associated morbidity and mortality [58]. Both daptomycin and linezolid nonsusceptible strains, which are considered first-line therapies, have been increasing in the USA primarily in immunocompromised and antibiotic-experienced patients [59,60]. Infections caused by E. faecium are typically of intra-abdominal, urinary, skin, or vascular access etiology. Before lefamulin can be employed as targeted therapy for resistant enterococcal infections, additional clinical and pharmacodynamic data would better facilitate the use of lefamulin for severe enterococcal infections. The Phase II ABSSSI study did not report outcomes related to isolation of E. faecium, and there are no published prospective or retrospective data on the topic to date [48]. Unfortunately, prospective randomized trials on severe $E$. faecium infections are logistically difficult to complete and it is unlikely to have such studies in the near future. With higher MIC distributions, the likelihood of target attainment based on thresholds using other organisms may be low. Readily accessible susceptibility testing in institutional microbiology labs would also be necessary, as susceptibility to other antimicrobial classes would not predict efficacy with lefamulin.

\section{Sexually Transmitted Infections: Neisseria gonorrhoeae and Mycoplasma genitalium}

Drug-resistant $N$. gonorrhoeae is considered an urgent threat by the CDC due to its ease of transmission, ability to develop resistance, and the complications associated with untreated infections [1]. Lefamulin exhibited minimal cross-resistance to gonococcus when macrolide-, quinolone-, and tetracyclinenon-susceptible isolates were tested, which can certainly be beneficial in the future scope of global and public health. The $\mathrm{MIC}_{50 / 90}$ against $251 \mathrm{~N}$. gonorrhoeae strains were $0.25 / 1 \mathrm{mg} /$ $\mathrm{L}$ and ranged from $0.004-2 \mathrm{mg} / \mathrm{L}$ [7]. In another in vitro analysis examining 25 strains, the $\mathrm{MIC}_{50 / 90}$ were $0.12 / 0.5 \mathrm{mg} / \mathrm{L}$. The activity of lefamulin was not impacted by inactivation of MacAB/NorM efflux pumps; however, a significant change in MIC (4-6-fold) was observed when MtrCDE efflux pump was inactivated [7]. Further data studying lefamulin's pharmacokinetics, pharmacodynamics, resistance, and clinical efficacy in the setting of MDR. $N$. gonorrhoeae infections are needed; there are no active clinical trials for lefamulin in the setting of sexually transmitted infection (STI).

Lefamulin is also active against M. genitalium, another STI implicated in the most recent issue of the CDC threats report $[1,61]$. In the presence of macrolide resistance, M. genitalium infections become very difficult to treat. Although the target binding site for both lefamulin and macrolides is the $50 \mathrm{~S}$ ribosome, lefamulin maintained potency against the five macrolide-resistant strains of $M$. genitalium with MICs ranging from 0.016 to $0.063 \mathrm{mg} / \mathrm{L}$. Another analysis examined 21 macrolide-resistant strains and observed similar potency and MIC range [8]. Lefamulin is also active against $C$. trachomatis and thus may be useful in co-infection [61].

\section{Specialized Populations}

\section{High-Risk/Recurrent Clostridiodes difficile Infections}

If over $50 \%$ of hospitalized patients receive an antimicrobial during hospitalization [62], and 1 in 5 exposed patients develop an antimicrobial-related ADE, in theory, $10 \%$ of all hospitalized adults suffer from an ADE [63]. This is likely underestimated, given that the lack of available data describing incidence/duration of microbiota disruption and its subsequent consequences - an $\mathrm{ADE}$ that is not easily measured in large cohorts. Clostridiodes difficile can be rare in patients otherwise at low risk, receiving broad-spectrum antibiotics, 
hence low incidence in LEAP-1 and LEAP-2. However, an often unmeasured, and more frequent antibiotic-related, ADE is the collateral damage that leads to colonization of C. difficile and resistant organisms. Restriction of oral antimicrobials commonly used for respiratory tract infections (i.e., amoxicillin-clavulanate, fluoroquinolones, clindamycin, cephalosporins) significantly reduces incidence of CDI [64]. Lefamulin could be considered to have a reduced amount of "collateral damage" compared to other commonly used oral antimicrobials. Lefamulin's spectrum of Gram-positive, atypical, and limited Gram-negative organisms is similar to tetracyclines, which as a class are far less implicated in CDI [61]. There were no significant differences in incidence of C. difficile or multi-drug-resistant organisms when compared to moxifloxacin, and interestingly, more diarrhea with oral lefamulin (but less with IV) [46, 47]. This could be advantageous if adopted as CABP therapy in comparison to other recommended agents. Thorough microbiome studies have yet to be conducted with lefamulin, and no antimicrobial is free of risk.

\section{A Steward's Perspective in a Looming Antimicrobial Crisis}

It remains unclear where the predominant uptake of systemic pleuromutilins will be. There have been numerous calls to action to bring antimicrobials with novel mechanisms of action to the market [65]. And despite achieving the goal of 10 new antimicrobials approved in the USA by 2020 as outlined in the Infectious Diseases Society of America $10 \times$ '20 initiative of 2010 [66], antibiotic development continues to be an uphill battle. Given that there are several readily available and less costly agents recommended by societal guidelines, the immediate and frequent use of lefamulin for more common indications such as CABP and ABSSSI in hospitalized adults is unlikely without significant reimbursements. Health systems are paid out on diagnosis-related group (DRG) models which do not specifically include antimicrobial expenditures. Thus, there are pressures that drive cost avoidance with antimicrobial use, which may not always be in the best interest of a patient [67]. Ambulatory patients with qualifying insurance plans may be a larger population of usage, pending the local availability and operationalization of additional steps such as prior authorization. Even with the availability of newer, safer, and more effective antimicrobials for MDR Gram-negative infections, the uptake of novel agents in the USA has been slow while systemic polymyxin use has not drastically decreased [68].

Lefamulin was granted Qualified Infectious Disease Product (QIDP) status by the FDA which allows 5 years of marketing exclusivity and eligibility for Fast Track designation $[69,70]$. However, the current pull incentives alone have not been able to keep the highest producers of new antimicrobials in the market. Clinical trials cost millions, and clinicians seek answers in patient populations that are difficult to identify and/or enroll, which requires robust multicenter studies that will ultimately span over years. Over 30 million United States dollars (USD) were provided from the Biomedical Advanced Research and Development Authority (BARDA) to support the development of meropenem/ vaborbactam, one of the two available beta-lactam betalactamase-inhibitor combinations available in the USA for carbapenemase-producing Enterobacterales (CRE) [71]. Achaeogen received almost US $\$ 150$ million from BARDA and the US Department of Health and Human Services to develop plazomicin, a unique aminoglycoside antibiotic that was added to the World Health Organization Essential Medicines List. These incentives were still inadequate due to low distribution volumes and price points for small patient populations, leading to both companies filing for bankruptcy $[67,72]$. Medications in the oncology space, such as avapritinib (Ayvakit $($ ), can be fast tracked and granted orphan drug status following small, open-label single-arm studies in patients with rare conditions; effectiveness against specific mutations can also expand use and indications [73]. Perhaps the market entry process for antimicrobials is overly rigorous, demanding large and costly randomized trials to evaluate endpoints in disease states, e.g., ABSSSI, urinary tract infections, rather than populations where the drug may benefit most, e.g., multi-drug resistance. Recently, the antiviral remdesivir was granted Orphan Drug status by the FDA for the management of SARS-CoV-2; however, the request was rescinded after criticisms of monopolizing the therapy [74].

Can pharmaceutical companies with a new antimicrobial such as lefamulin succeed in an era of intense antimicrobial stewardship and costs-savings initiatives? A bill was put forward in 2018 excluding antimicrobials from the DRG. The act, DISARM (Developing an Innovative Strategy for Antimicrobial Resistant Microorganisms Act of 2019), would allow add-on payments to hospitals enrolled with Medicare using a qualified agent beginning in 2020 [75]. Others describe the development model as ineffective and suggest a path involving non-profit organizations to promote antibiotic R\&D and avoid the pressures of higher profit margins [9]. Some have even pointed towards stewards, physicians, and pharmacies as those among other responsible parties who "killed antibiotic development" $[72,76]$. Regardless, as responsible clinicians and prescribers, prompt initiation of optimal medication should be a priority. While an agent with a novel mechanism such as lefamulin should be protected, stewardship involves identification of the populations that would benefit most, such as those with severe intolerances, historical C. difficile, or multi-drug-resistant infections. 


\section{Conclusion}

Lefamulin is a pleuromutilin antibiotic that inhibits protein synthesis inhibition and has a spectrum of activity that includes most Gram-positive and atypical organisms, H. influenzae, M. catarrhalis, and some pertinent anaerobes. Lefamulin has been shown to be an effective option in treating CABP, and other Phase II studies suggest efficacy in treating ABSSSI. Until more appropriate reimbursement models are developed, we suspect that health systems will continue to utilize existing, generic antibiotics (i.e., beta-lactams \pm macrolide/tetracycline, or fluoroquinolones) over newly developed agents for treatment of CABP and ABSSSI due to cost and lack of presence in treatment guidelines. Populations who may benefit from lefamulin over traditional therapies include those in settings with a high prevalence of communityassociated MRSA, patients at higher risk of adverse effects from fluoroquinolone use, and in patients with history of $C$. difficile or multiple antimicrobial intolerances. Interesting opportunities for use also exist in multi-drugresistant Gram-positive and sexually transmitted organisms, although we are unlikely to see clinical trial data in these populations soon and will await real-world experiences. While a hospital's and patient's ability to financially cope and comply with therapy must always be considered, cost alone should not dictate treatment. We operate in an era where the development of antimicrobials is undesirable due to the lack of profitability and have witnessed the disappearance of multiple agents and manufacturers. Lefamulin is a well-tolerated agent with a novel mechanism, availability in both IV and PO formulations, and it has been rigorously studied for safety and efficacy for CABP.

Authors' Contributions NJM and MPV both contributed equally to the design and writing of this manuscript.

Data Availability Not applicable.

\section{Compliance with Ethical Standards}

Conflict of Interest MPV has received grant funding from Paratek Pharmaceuticals.

Ethics Approval and Consent to Participate Not applicable.

Consent for Publication Not applicable.

Code Availability Not applicable.

Human and Animal Rights and Informed Consent This article does not contain any studies with human or animal subjects performed by any of the authors.

\section{References}

1. Centers for Disease Control and Prevention. Antibiotic Resistance Threats in the United States, 2019. Accessed 17 January 2020. Available at: https://www.cdc.gov/drugresistance/pdf/threatsreport/2019-ar-threats-report-508.pdf

2. Spellberg B, Bartlett J, Wunderink R, Gilbert DN. Novel approaches are needed to develop tomorrow's antibacterial therapies. Am J Respir Crit Care Med. 2015;191(2):135-40.

3. Centers for Disease Control and Prevention. The core elements of hospital antibiotic stewardship programs: 2019. Accessed 27 January 2020. Available at: https://www.cdc.gov/antibiotic-use/ healthcare/pdfs/hospital-core-elements-H.pdf

4. Barlam TF, Cosgrove SE, Abbo LM, MacDougall C, Schuetz AN, Septimus EJ, et al. Implementing an antibiotic stewardship program: guidelines by the Infectious Diseases Society of America and the Society for Healthcare Epidemiology of America. Clin Infect Dis. 2016 May 15;62(10):e51-77.

5. Paukner S, Riedl R. Pleuromutilins: potent drugs for resistant bugsmode of action and resistance. Cold Spring Harb Perspect Med. 2017;7:a027110.

6. Lefamulin (XENLETA) Package Insert. Accessed 5 February 2020. Available at: https://www.accessdata.fda.gov/drugsatfda docs/label/2019/211672s000,211673s000lbl.pdf

7. Jacobsson $\mathrm{S}$, Paukner $\mathrm{S}$, Golparian $\mathrm{D}$, et al. In vitro activity of the novel pleuromutilin lefamulin (BC-3781) and effect of efflux pump inactivation on multidrug-resistant and extensively drug-resistant Neisseria gonorrhoeae. Antimicrob Ag Chemother. 2017;61(11): e01497-17.

8. Jensen JS, Paukner S. Lefamulin is highly active in vitro against multi-drug resistant mycoplasma genitalium strains. 27th European Clinical Congress on Microbiology and Infectious Diseases. Vienna, Austria.

9. Nielsen TB, Brass EP, Gilbert DN, Bartlett JG, Spellberg B. Sustainable discovery and development of antibiotics - is a nonprofit approach the future? N Engl J Med. 2019;381(6):503-5.

10. 2019 Antibacterial agents in clinical development: an analysis of the antibacterial clinical development pipeline. Geneva: World Health Organization; 2019. Licence: CC BY-NC-SA 3.0 IGO.

11. Novak R, Shlaes DM. The pleuromutilin antibiotics: a new class for human use. Curr Opin Investig Drugs. 2010;11(2):182-91.

12. Novak R. Are pleuromutilin antibiotics finally fit for human use? Ann N Y Acad Sci. 2011;1241:71-81.

13. Nabriva Therapeutics. News \& Publications. Accessed 1 February 2020. Available at: https://www.nabriva.com/news

14. Eyal Z, Matzov D, Krupkin M, Wekselman I, Paukner S, Zimmerman E, et al. Structural insights into species-specific features of the ribosome from the pathogen Staphylococcus aureus. Proc Natl Acad Sci U S A. 2015;112(43):E5805-14.

15. Mendes RE, Paukner S, Doyle TB, Gelone SP, Flamm RK, Sader HS. Low Prevalence of Gram-Positive Isolates Showing Elevated Lefamulin MIC Results during the SENTRY Surveillance Program for 2015-2016 and Characterization of Resistance Mechanisms. Antimicrob Agents Chemother. 2019;63(4):e02158-18.

16. FDA Identified Interpretive Criteria. Lefamulin oral and injection products. Food and Drug Administration Accessed 20 March 2020. Available at: https://www.fda.gov/drugs/development-resources/ lefamulin-oral-and-injection-products

17. Wicha WW, Prince WT, Lell C, Heilmayer W, Gelone SP. Pharmacokinetics and tolerability of lefamulin following intravenous and oral dosing. J Antimicrob Chemother. 2019;74(Supplement_3):iii19-26. https://doi.org/10.1093/jac/ dkz087. 
18. Paukner S, Gelone SP, Arends SJR, et al. Antibacterial activity of lefamulin against pathogens commonly causing community acquired bacterial pneumonia: SENTRY antimicrobial surveillance program (2015-2016). AAC. 2019;63(4):e02161-18.

19. Pfaller MA, Huband MD, Shortridge D, et al. Surveillance of Omadacycline activity tested against clinical isolates from the United States and Europe as part of the 2016 SENTRY antimicrobial surveillance program. AAC. 2018;62(4):e02327-17.

20. Flamm RK, Rhomberg PR, Huband MD, Farrell DJ. In vitro activity of delafloxacin tested against isolates of Streptococcus pneumoniae, Haemophilus influenzae, and Morazella catarrhalis. AAC. 2016;60(10):6381-6.

21. Kohlhoff SA, Huerta N, Hammerschlag MR. In vitro activity of omadacycline against Chlamydia pneumoniae. AAC. 2019;63(2): e01907-18.

22. Waites KB, Crabb DM, Liu Y, et al. In vitro activities of Omadacycline (PTK 0796) and other antimicrobial agents against human mycoplasmas and Ureaplasmas. AAC. 2016;60(12):75025.

23. Villano S, Steenbergen J, Loh E. Omadacycline: development of a novel aminomethylcycline antibiotic for treating drug-resistant bacterial infections. Future Microbiol. 2016;11(11):1421-34.

24. Schulin T, Wennersten CB, Ferraro MJ, et al. Susceptibilities of Legionella spp. to newer antimicrobials in vitro. AAC. 1998;42(6):1520-3.

25. Pereyre $\mathrm{S}$, Renaudin $\mathrm{H}$, Bebear $\mathrm{C}$, et al. In vitro activities of the newer quinolones garenoxacin, gatifloxacin, and Gemifloxacin against human mycoplasmas. AAC. 2004;48(8):3165-8.

26. Pfaller MA, Sader HS, Rhomberg PR, et al. In vitro activity of delafloxacin against contemporary bacterial pathogens from the United States and Europe, 2014. AAC. 2017;61(4):e02609-16.

27. Pfaller MA, Rhomberg PR, Huband MD, Flamm RK. Activity of omadacycline tested against Streptococcus pneumoniae from a global surveillance program (2014). Diagn Microbiol Infect Dis. 2018;90:143-7.

28. Hammerschlag MR. Activity of trimethoprim-sulfamethoxazole against Chlamydia trachomatis in vitro. Rev Infect Dis. 1982;4: $500-5$.

29. Lawrence L, Danese P, DeVito J, Franceschi F, Sutcliffe J. In vitro activities of the rx- 01 oxazolidinones against hospital and community pathogens. AAC. 2008;52(5):1653-62.

30. Jacobs JA, Stobberingh EE. In-vitro antimicrobial susceptibility of the 'Strepcoccus milleri' group (Streptococcus anginosus, Streptococcus constellatus, and Streptococcus intermedius). JAC. 1996;37:371-5.

31. Grayson ML. Kucers' the use of antibiotics: a clinical review of antibacterial, antifungal, antiparasitic and antiviral drugs. 7th ed. London: Hodder Arnold; 2017.

32. The European Committee on Antimicrobial Susceptibility Testing. MIC- and inhibition zone diameter distributions of microorganisms without and with resistance mechanisms, version 5.26. Accessed 22 January, 2020. Available at: https://mic.eucast.org/Eucast2/

33. Bhavnani SM, Zhang L, Hammel JP, et al. Pharmacokinetic/ pharmacodynamic target attainment analyses to support intravenous and oral lefamulin dose selection for the treatment of patients with community-acquired bacterial pneumonia. J Antimicrob Chemother. 2019;74(Supplement_3):iii35-41.

34. Zeitlinger M, Schwameis R, Burian A, Burian B, Matzneller P, Müller M, et al. Simultaneous assessment of the pharmacokinetics of a pleuromutilin, lefamulin, in plasma, soft tissues, and pulmonary epithelial lining fluid. J Antimicrob Chemother. 2016;71(4): 1022-6.

35. Wicha WW, Strickmann DB, Paukner S. Pharmacokinetics/ pharmacodynamics of lefamulin in a neutropenic murine pneumonia model with Staphylococcus aureus and Streptococcus pneumoniae. J Antimicrob Chemother. 2019;74(Supplement_3): iii11-8.

36. Valcke Y, Pauwels R, Van der Straeten M. Pharmacokinetics of antibiotics in the lungs. Eur Respir J. 1990;3(6):715-22.

37. Schmidt U, Wicha WW, Obermayr F, et al. BC-3781: evaluation of the CYP3A interaction potential. 21st European Congress of Clinical Microbiology and Infectious Diseases 2011; Abstract P1524.

38. NDA/BLA Multi-disciplinary Review and Evaluation \{NDA 211672 and NDA 211673\} XENLETA / lefamulin injection and tablets. Food and Drug Administration. Accessed 20 March 2020. Available at: https://www.accessdata.fda.gov/drugsatfda_docs/nda/2019/ 211672Orig1s000,\%20211673Orig1s000MultidisciplineR.pdf

39. Wicha WW, Ivezic-Schoenfeld Z, Novak R. Pharmacokinetic, mass balance and tissue distribution of [14C]-BC-3781 in nonpigmented rats. 20th European Congress of Clinical Microbiology and Infectious Diseases 2010; Abstract P909.

40. Wicha WW, Marbury TC, Dowell JA, et al. Pharmacokinetics (PK) and safety of lefamulin (LEF) after single intravenous dose administration in subjects with impaired renal function and in those requiring hemodialysis. Open Forum Infect Dis. 2019;6(Supplement 2):S318.

41. Craig WA, Andes D, Ivezic-Schoenfeld Z, et al. In vivo pharmacodynamic activity of BC-3781. 20th European Congress of Clinical Microbiology and Infectious Diseases 2010; Abstract P907.

42. Wicha WW, Fischer E, Kappes B, et al. Comparative pharmacodynamics of BC-3781 in murine Streptococcus pneumoniae thigh and lung infection models. 53rd Interscience Conference on Antimicrobial Agents and Chemotherapy 2013; Abstract A-013.

43. Wicha WW, Paukner S, Strickmann DB, et al. Pharmacokineticspharmacodynamics of lefamulin in a neutropenic murine lung infection model. Interscience Conference on Antimicrobial Agents and Chemotherapy/International Congress of Chemotherapy and Infection 2015; Abstract A-037.

44. Zhang L, Bhavnani SM, Ambrose PG, et al. Population pharmacokinetic (PPK) analysis for lefamulin using phase 1 data and assessment of optimal PK sampling strategies (OSS) for a phase 3 community-acquired bacterial pneumonia (CABP)study. ID Week 2016; Abstract 1944.

45. Veve MP, Wagner JL. Lefamulin: review of a promising novel pleuromutilin antibiotic. Pharmacotherapy. 2018 Sep;38(9):93546.

46. ORIGINAL File TM, Goldberg L, Das AF, Saviski J, et al. Efficacy and safety of intravenous to oral lefamulin, a pleuromutilin antibiotic, for the treatment of community acquired bacterial pneumonia: the phase III lefamulin evaluation against pneumonia (LEAP 1) trial. Clin Inf Dis. 2019;69(11):1856-67.

47. Alexander E, Goldberg L, Das AF, Moran GJ, Sandrock C, Gasink $\mathrm{LB}$, et al. Oral lefamulin vs moxifloxacin for early clinical response among adults with community acquired bacterial pneumonia: the LEAP-2 randomized clinical trial. JAMA. 2019;322(17):1661-71.

48. Prince WT, Ivezic-Schoenfeld Z, Lell C, Tack KJ, Novak R, Obermayr F, et al. Phase II clinical study of BC-3781, a pleuromutilin antibiotic, in treatment of patients with acute bacterial skin and skin structure infections. Antimicrob Agents Chemother. 2013;57(5):2087-94.

49. Wicha WW, Lell C, Obermayr F, et al. An age and gender study investigating the safety, tolerance and pharmacokinetics of BC3781. 50th Interscience Conference on Antimicrobial Agents and Chemotherapy 2010; Abstract A1-019.

50. Lakhundi S, Zhang K. Methicillin-resistant Staphylococcus aureus: molecular characterization, evolution, and epidemiology. Clin Microbiol Rev. 2018;12:31(4).

51. Parikh MP, Octaria R, Kainer MA. Methicillin-resistant Staphylococcus aureus bloodstream infections and injection drug 
use, Tennessee, USA, 2015-2017. Emerg Infect Dis. 2020;26(3): 446-53.

52. Wicha WW, Ivezic-Schoenfeld Z, Novak R. Pre-clinical efficacy of BC-3781 in thigh and bacteremia infections caused by staphylococci. $50^{\text {th }}$ Interscience Conference on Antimicrobial Agents and Chemotherapy 2010; Abstract F1-2109.

53. Fischer E, Kappes BC, Wicha WW. Efficacy of lefamulin against Staphylococcus aureus - induced bacteremia in a neutropenic and immunocompetent murine model. ID Week 2017; Abstract 1509.

54. Li HK, Rombach I, Zambellas R, Walker AS, McNally M, Atkins $\mathrm{BL}$, et al. Oral versus intravenous antibiotics for bone and joint infection. N Engl J Med. 2019;380(5):425-36.

55. Jorgensen SCJ, Lagnf AM, Bhatia S, Shamim MD, Rybak MJ. Sequential intravenous-to-oral outpatient antibiotic therapy for MRSA bacteraemia: one step closer. J Antimicrob Chemother. 2019;74(2):489-98.

56. Willekens R, Puig-Asensio M, Ruiz-Camps I, Larrosa MN, González-López JJ, Rodríguez-Pardo D, et al. Early oral switch to linezolid for low-risk patients with Staphylococcus aureus bloodstream infections: a propensity-matched cohort study. Clin Infect Dis. 2019;69(3):381-7.

57. Iversen K, Ihlemann N, Gill SU, Madsen T, Elming H, Jensen KT, et al. Partial oral versus intravenous antibiotic treatment of endocarditis. N Engl J Med. 2019;380(5):415-24.

58. Mercuro NJ, Davis SL, Zervos MJ, Herc ES. Combatting resistant enterococcal infections: a pharmacotherapy review. Expert Opin Pharmacother. 2018;19(9):979-92.

59. DiPippo A, Tverdek FP, Tarrand JJ, et al. Daptomycin nonsusceptible Enterococcus faecium in leukemia patients: role of prior daptomycin exposure. J Inf Secur. 2017;74(3):243-7.

60. Greene MH, Harris BD, Nesbitt WJ, et al. Risk factors and outcomes associated with acquisition of daptomycin and linezolidnonsusceptible vancomycin-resistant Enterococcus. Open Forum Infect Dis. 2018;5(10):ofy185.

61. Paukner S, Gruss A, Jensen JS. In vitro activity of lefamulin against sexually transmitted bacterial pathogens. Antimicrob Ag Chemother. 2018;62(5):e02380-17.

62. Baggs J, Fridkin SK, Pollack LA, et al. Estimating national trends in inpatient antibiotic use among US hospitals from 2006 to 2012. JAMA Intern Med. 2016;176(11):1639-48.

63. Tamma PD, Avdic E, Li DX, Dzintars K, Cosgrove SE. Association of Adverse Events With Antibiotic Use in Hospitalized Patients. JAMA Intern Med. 2017;177(9):1308-1315.

64. Lawes T, Lopez-Lozano JM, Nebot CA. Effect of a national 4C antibiotic stewardship intervention on the clinical and molecular epidemiology of Clostridium difficile infections in a region of Scotland: a non-linear tie-series analysis. Lancet Infect Dis. 2017;17(2):194-206.

65. Shales DM. The economic conundrum for antibacterial drugs. Antimicrob Ag Chemother. 2020;64(1):e02057-19.

66. Gilbert DN, Guidos RJ, Boucher HW, et al. The $10 \mathrm{x}$ '20 initiative: pursuing a global commitment to develop 10 new antibacterial drugs by 2020. Clin Infect Dis. 2010;50(8):1081-3.
67. Blewett M, Kocher B, Shady B. How to cure the antibiotic industry's profitability infection. 14 Nov, 2019. Commentary, Antibiotics. Accessed 11 Jan 2020. Available at: https://fortune. com/2019/11/14/antibiotics-funding-achaogen-tetraphasepharmaceuticals/

68. Clancy CJ, Potoski BA, Buehrle D, Nguyen MH. Estimating the treatment of carbapenem-resistant Enterobacteriaceae infections in the United States using antibiotic prescription data. Open Forum Inf Dis. 2019;6(8):ofz344 (1-8).

69. Qualified infectious disease product designation questions and answers guidance for industry. January 2018. U.S. Department of Health and Human Services. Food and Drug Administration. Center for Drug Evaluation and Research. Silver Spring, MD.

70. $112^{\text {th }}$ Congress. $1^{\text {st }}$ Session. H.R. 2182. Sec 505E. Extension of exclusivity period for new qualified infectious diseases products. June 15, 2011. Available at: https://www.congress.gov/bill/112thcongress/house-bill/2182/text

71. BuisnessWire.com. The Medicines Company announces TANGO2 trial of meropenem-vaborbactam (formerly, Carbavance) stopped early for superior benefit-risk compared to best available therapy for CRE. 2017. Accessed 14 Feb 2020. Available at: https://www. businesswire.com/news/home/20170725005394/en/MedicinesCompany-announces-TANGO-2-trial-meropenem-vaborbactamCarbavance

72. Jacobs A. Crisi looms in antibiotics as drug makers go bankrupt. Deadly Germs, Lost Cures. The New York times. 26 December, 2019. Accessed 11 January, 2020. Available at: https://www. nytimes.com/2019/12/25/health/antibiotics-new-resistance.html? auth=link-dismiss-google1 tap

73. FDA approves avapritinib for gastrointestinal stromal tumor with a rare mutation. Resources for Information on Approved Drugs. 9 January, 2020. Accessed 11 January, 2020. Available at: https:// www.fda.gov/drugs/resources-information-approved-drugs/fdaapproves-avapritinib-gastrointestinal-stromal-tumor-rare-mutation

74. Gilead Sciences Statement on Request to Rescind Remdesivir Orphan Drug Designation. Gilead Sciences, Inc. Accessed 20 March 2020. Available at: https://www.gilead.com/news-andpress/company-statements/gilead-sciences-statement-on-requestto-rescind-remdesivir-orphan-drug-designation

75. Morales J. DISARM Act provides framework needed to spur antibiotic R\&D, protect existing drugs. 5 June, 2019. News Release. Accessed 11 January, 2020. Available at: https://www.idsociety. org/news\%2D\%2Dpublications-new/articles/2019/disarm-actprovides-framework-needed-to-spur-antibiotic-rd-protect-existingdrugs/

76. Boucher $\mathrm{H}, \mathrm{Yu} \mathrm{C}$. We're part of the problem: how ID killed antibiotic development. IDWeek 2019. Conference Proceedings. Washington DC, USA. 3 October, 2019.

Publisher's Note Springer Nature remains neutral with regard to jurisdictional claims in published maps and institutional affiliations. 